\title{
WHO ARE THE USERS OF SPEED REGULATION ASSISTANCE? COMPARING DRIVER CHARACTERISTICS OF CASUAL AND INTENSIVE SYSTEM USERS
}

\author{
Véronique Huth, Corinne Brusque, Marie-Pierre Bruyas \& Valérie Lancelle \\ IFSTTAR (LESCOT), Université de Lyon \\ Lyon, France \\ Email: veronique.huth@ifsttar.fr
}

\begin{abstract}
Summary: Speed regulation assistance can contribute to road safety provided that drivers use the systems on a regular basis. With the objective to gain knowledge about drivers who use Cruise Control and the Speed Limiter, a comparison of the characteristics of casual and intensive users was performed with survey data. The results show that gender and annual mileage play a role for the usage frequency of Cruise Control, whereas the usage frequency of the Speed Limiter depends on age. Consistent effects of the car use for business matters and the use of other invehicle technologies were found on the usage frequency of both systems. The predominant motive to reduce speeding found for both systems corresponds with the objective of speed regulation assistance as a safety measure. It was complemented with a comfort benefit perceived by Cruise Control users.
\end{abstract}

\section{INTRODUCTION}

Speed plays a major role in road safety. As the World Report on Road Traffic Injury Prevention points out, speed influences both crash occurrence and crash severity (Peden et al., 2004). Even small decrements in traffic speeds lead to a significant increase in road safety because road trauma is reduced (e.g. Young \& Regan, 2006). Correspondingly, the OECD recommends safety interventions to reduce speeding behavior as one essential road safety target in its report on a Safe System approach, aiming at avoiding fatalities or injuries in the event of a crash (ITF, 2008). In addition to the implementation of appropriate speed enforcement strategies, the report suggests to promote the use of new technologies that regulate driving behavior. Previous research has shown that speed regulation assistance can decrease the probability and severity of crashes (Young \& Regan, 2006). A review of studies on Cruise Control revealed that the system had a positive impact on travel speeds, speed variability and traffic flow (van Kampen, 1996; cited in Young \& Regan, 2006). Similarly speed controls systems that limit the driving speed have proven to reduce speed peaks and speed variation (Várheli \& Mäkinen, 2001). Accordingly, a widespread use of these systems would considerably reduce crashes, given that it would provoke a decrease in braking and maneuvers.

Nowadays, speed regulation systems have become mature technologies in Europe, i.e. they are available on the market and are already adopted by a considerable share of drivers (Bonnard et al., 2009). This paper deals with two of those technologies:

- Cruise Control (CC) automatically controls the driving speed at a level chosen by the driver. With conventional CC, the driver has to brake in order to decelerate the vehicle and to overrule the throttle control in order to increase the speed. Adaptive Cruise Control (ACC) maintains the preset speed only as long as the traffic conditions allow for 
it and slows down the vehicle when approaching another vehicle based on its detection by a radar or laser setup.

- The Speed Limiter (SL) sets the vehicle's maximum speed. The driver will not be able to accelerate the vehicle above the set speed, unless he uses the kick-down function.

In Europe's most sold vehicles, a relatively low level of availability has been identified for ACC, whereas conventional CC and SL have been assigned much higher availability ratings (Bonnard et al., 2009). Paradoxically, most studies on speed regulation assistance focus on ACC and far less is known on the use of CC and SL yet (Young \& Regan, 2006; Vollrath et al., 2011). Studies by Lheureux et al. (2006) have dealt with drivers' decision to use or not to use CC and SL. They have analyzed the influence of external conditions, such as the road type, the traffic condition and weather, on the usage frequency of CC and SL. Furthermore, age and gender have been identified as relevant static dimensions, but a more extensive survey data collection with the aim to quantify relevant variables that influence system use has been claimed.

A report on the use of intelligent systems in vehicles, published by the European Commission, argues that gender, age, car type and annual mileage play a role in this context (EC, 2006). Given that the safety benefit of speed regulation assistance depends on the extent of its use, it is not only important to know who the users of those technologies are but also which characteristics allow distinguishing casual users from intensive users. For this reason, the present paper focusses on users of speed regulation assistance and describes their main characteristics, comparing casual and intensive users of CC and SL.

\section{METHOD}

This paper analyzes questionnaire data, which were gathered as part of a broader survey on the use of in-vehicle technologies conducted in the framework of the INTERACTION project (www.interaction-fp7.eu). In order to pursue the objective of this paper, a sub-set of French data containing only those drivers who indicated to be using CC and/or SL was selected.

\section{Survey}

The survey conducted with INTERACTION consisted in a standardized web-based questionnaire. It was carried out by a polling company using an internet panel. Given that one aim was to explore whom the users of in-vehicle technologies are, the characteristics of the target sample were not pre-defined and the sample is thus not meant to be representative of the French driver population. The use of at least one in-vehicle technology (speed regulation system or navigation system) was a screening question, including only users into the sample. This procedure allows for a more detailed and meaningful description of the users than it would be possible in a broader sample, which is representative of the population but where users only constitute a small share of respondents. Further details on the INTERACTION survey can be found in Britschgi and Rämä (2010).

\section{Sample}

From the original sample of $\mathrm{N}=1177$ French respondents, the present study analyzed the data set with the $N=474$ speed regulation assistance users. This sample consisted of $n=245$ exclusive CC 
users, $n=30$ exclusive SL users and n=199 driver who use both systems. Figure 1 shows the usage frequencies indicated by the drivers. A median-split performed on this variable leads to the distinction of the following two user groups for both systems:

- casual users, who use the system rarely (at least once a year) or occasionally (at least once a month)

- $\quad$ intensive users, who use the system regularly (at least once a week) or frequently (at least once a day).

The distributions indicate a greater popularity of Cruise Control, with a higher absolute number of users in the sample and a somewhat higher share of intensive users than for the Speed Limiter (Figure 1). The car types used by the respondents could only be identified for about two thirds of the sample due to unspecific answers. The car categories are therefore not included as a variable into the analyses, but their distribution is shown in Figure 2 on a descriptive level. For both systems, it appears that intensive users are more frequently represented as medium size car or multi-purpose-vehicle (minivan) drivers, whereas the share of small car drivers is greater for casual users than for intensive users.



Figure 1. Distribution of usage frequency Figure 2. Distribution of car categories of casual vs. intensive CC for Cruise Control users and SL users (left) and SL users (right)

\section{Parameters and Analysis}

Table 1 shows the parameters that have been used so as to characterize the users of CC and SL. Comparisons between the casual and intensive users have been carried out by separate statistical testing for CC and SL. For dichotomous variables Chi-Square tests were performed and the distributions of ordinal variables were compared by means of Mann-Whitney-U-tests.

Table 1. Variables used in the analysis

\begin{tabular}{ll}
\hline Parameter & Scale \\
\hline Frequency of system use & Casual [rarely, occasionally] vs. intensive [regularly, frequently] \\
\hline Gender & Male vs. female \\
Age & Four categories [18-29, 30-39, 40-49, 50+]
\end{tabular}


Km driven within the past 12 months

Car use also for business/professional matters

Frequency of other system use [CC, SL, Navigation System]

Main perceived benefit (first or second choice)
Three categories [<10 000 km, 10000 - 20000 km, > 20000 km]

Yes vs. No

Casual [rarely, occasionally] vs. intensive [regularly, frequently]

Five suggested categories [control speed, reduce speeding, improve comfort, improve safety, reduce fuel consumption]

\section{RESULTS}

Compared to the gender distribution in the French driver population (48\% male, 52\% female; source: www.insee.fr), the survey contained a higher proportion of male CC users (57\%) and male SL users (60\%, Figure 3). Statistical testing shows that male users were more represented as intensive CC users (61\%) than casual CC users (51\%, $\left.\chi^{2}(1)=4.152 ; p=.042\right)$. This effect could not be found in the comparison between intensive and casual SL users.

The distribution of age categories in the CC and SL user sample (Figure 4) also differed from what could be expected in the French driver population, where roughly one fifth corresponds to each of the first three age categories and two fifth represent the 50+ drivers (source:

www.insee.fr). Younger drivers (up to 40 years of age) seem to be overrepresented as speed regulation system users.

The comparison of the age distribution of casual and intensive CC users does not reveal any significant differences. For SL users, an age effect was detected as a tendency (Mann-Whitney$\mathrm{U}$-Test, $\mathrm{p}=.07$ ), with higher age categories having higher chances of being intensive users (Figure 4).

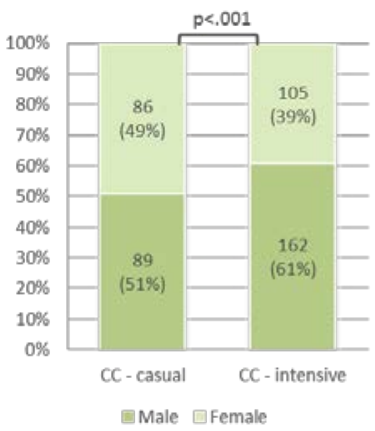

Figure 3. Gender distribution of casual vs. intensive CC (left) and SL users (right)

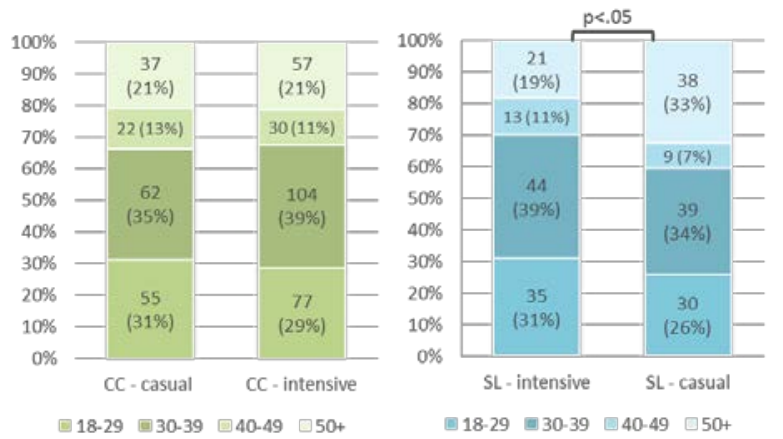

Figure 4. Distribution of age categories of casual vs. intensive CC (left) and SL users (right)

The mileage driven within the past 12 months proved to differ systematically between casual and intensive CC users (Mann-Whitney U test, $\mathrm{p}=.000$ ). Intensive CC use is related to higher annual mileage than casual CC use (Figure 5). No such effect was detectable among SL users. As illustrated in Figure 6, those drivers who indicated using their car also for business or professional matters were found to be overrepresented in the intensive user group for CC $\left(\chi^{2}(1)=9.283 ; p=.002 ; 35 \%\right.$ of intensive users vs. $22 \%$ of casual users). A significant effect was found in the same direction for SL $\left(\chi^{2}(1)=3.808\right.$; $p=.05$; $38 \%$ of intensive users vs. $26 \%$ of casual users). 


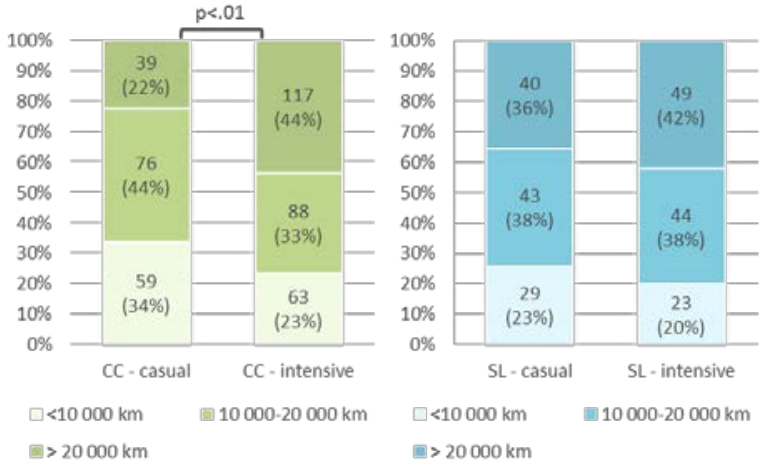

Figure 5. Distribution of annual mileage: casual vs. intensive CC (left) and SL users (right)

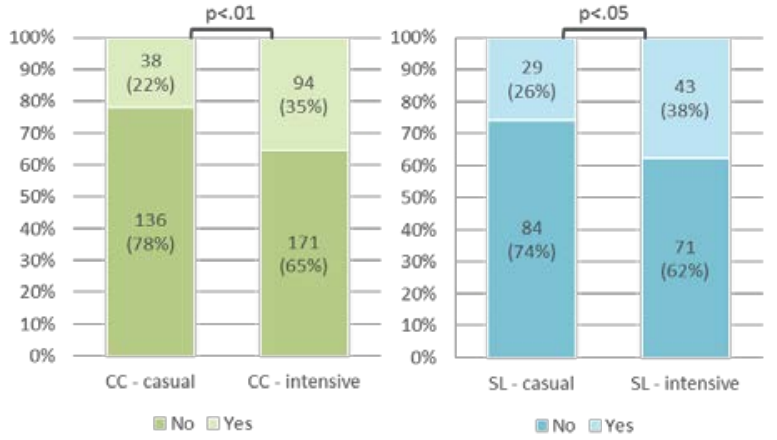

Figure 6. Distribution of car use for casual vs. intensive CC (left) and SL users (right)

The majority of CC users ( $n=245)$ indicated not to use the SL. Among the $n=72$ casual CC users who also use SL 72\% were casual SL users, whereas intensive CC users who use both systems $(n=127)$ were also intensive SL users at $66 \%$. Statistical testing confirms that intensive CC users are more likely than casual CC users to be intensive rather than casual users of SL $\left(\chi^{2}(1)=27.107\right.$; $\mathrm{p}=.000)$. Contrary to CC, SL is used exclusively only by a small share of users $(n=30)$. The group of casual SL users who indicated using CC too $(n=95)$ included 55\% casual CC users. Against that, out of the $n=104$ intensive SL users who also used CC $81 \%$ were intensive users of that system as well. Thus, intensive SL users are overrepresented as intensive CC users, as compared to casual SL users who rather tend to be casual CC users $\left(\chi^{2}(1)=27.107 ; p=.000\right)$.

The usage frequency of the Navigation System corresponds with effects on the use of speed regulation assistance described above. $\mathrm{N}=99$ CC users and $n=39$ SL users indicated not to use any Navigation System. Among the users of speed regulation and navigation ( $\mathrm{n}=127$ casual and $\mathrm{n}=218$ intensive CC, $\mathrm{n}=95$ casual and $\mathrm{n}=95$ intensive SL), $68 \%$ of casual CC users were casual Navigation System users and 51\% of intensive CC users were intensive Navigation System users. On the other hand, 67\% of casual SL users were casual Navigation System users and 61\% of intensive SL users were also intensive Navigation System users. Accordingly, the comparison of Navigation System usage frequencies reveals that significantly more intensive than casual CC users are intensive Navigation System users $\left(\chi^{2}(1)=11.305 ; \mathrm{p}=.001\right)$, just as intensive SL users are more likely to be intensive Navigation System users than casual SL users do $\left(\chi^{2}(1)=15.409\right.$; $\mathrm{p}=.000)$.

Finally, Figure 7 shows the main perceived benefits of the system use, which have been chosen as first or second most important benefit among the five suggested categories by the CC and SL users. The most frequently chosen benefit is the reduction of speeding, with particularly high percentages for SL. Furthermore, the distribution reflects that CC has a pronounced comfort aspect, which hardly appears for SL. Relatively few drivers considered the improvement of safety or a reduction of fuel consumption as a main benefit of the systems. However, the comparison of casual and intensive CC users reveals differences here. Intensive CC users were more likely to choose the improvement of safety than casual CC users $\left(\chi^{2}(1)=3.289 ; p=.001\right)$ and casual users were more likely to indicate a reduction of fuel consumption as a main benefit than intensive users $\left(\chi^{2}(1)=-2.005 ; p=.045\right)$. No differences between the choices of casual and intensive system users were detected for the other benefits of CC and for SL in general. 


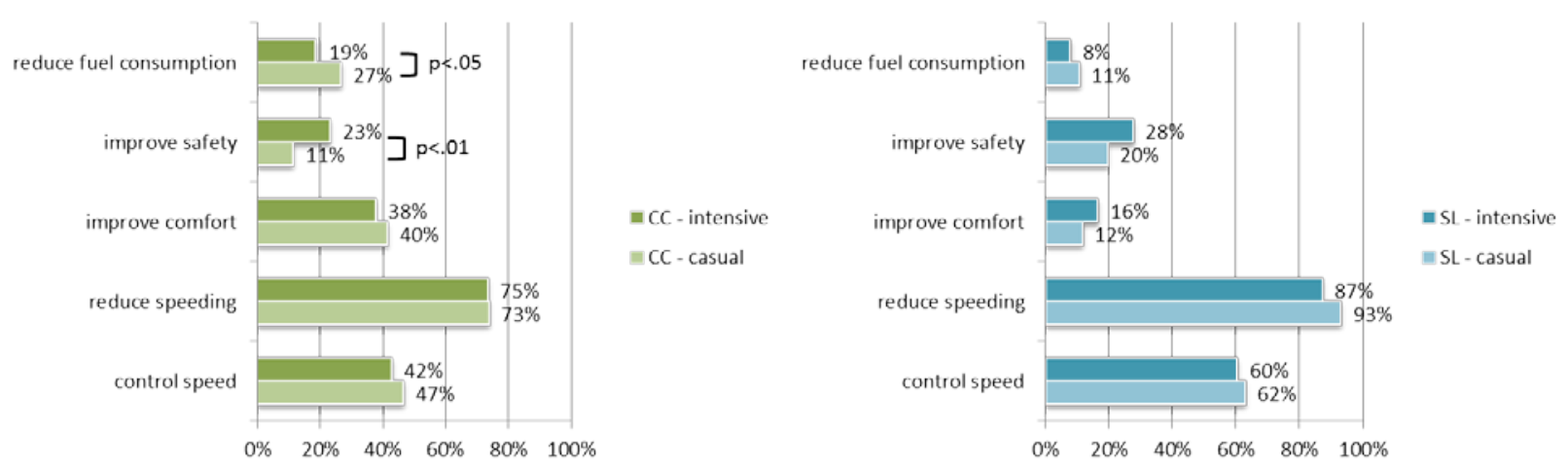

Figure 7. Distribution of main perceived benefits (chosen as first or second most important) of the system usage comparing intensive and casual users. CC (left) and SL users (right)

\section{CONCLUSION}

The analysis of driver characteristic of speed regulation assistance users presented in this paper allowed to gain relevant knowledge on the aspects that differentiate casual and intensive users of the systems, and to conclude if these characteristics are equally important for two different speed regulations systems.

The higher number of CC users as compared to SL users in the study sample corresponds with earlier results on drivers' preference of the CC compared to the SL (Pereira et al., in press). In France, cars are often simultaneously equipped with the CC and SL, mostly sharing one user interface. This explains the considerable share of mixed CC and SL users.

The frequency of CC use was associated to gender (more male intensive users) and the mileage driven during the past 12 months (more intensive users with higher annual mileage), whereas intensive SL usage showed to be more related to age. Although both casual and intensive SL users were relatively young in the majority (as did CC users), drivers in the higher age categories tended to be intensive SL users. Clear effects on the usage frequency of both systems have been found related to the car use for business purposes and to the frequency of the use of other invehicle technologies (SL or CC respectively, Navigation System). The latter might be due to a greater affinity for technology use or a better acceptance of assistance systems of those drivers. The main benefit attributed to both speed regulation systems by most participants, i.e. the reduction of speeding, corresponds with the OECD recommendation to promote the use of invehicle technologies for this purpose. Besides, CC is also considered as a comfort system, a result already found in focus group interviews (Pereira et al., in press). The distribution found in the present study confirms that CC is more associated with comfort than SL, and that SL in turn is more linked to the avoidance of speeding (c.f. Young \& Regan, 2006). The choice of the safety benefit by intensive users could be due to the experience these users have had with the systems. They might have passed a threshold of system understanding and trust that casual users have not reached yet. Similarly, Lheureux et al. (2006) reported positive perceptions of the CC and SL by long-term system owners.

A constraint of the present study is the quantification of system usage by the frequency rather than the mileage driven with assistance. This shortcoming is due to the difficulty of subjective estimations on distance-related usage, which are likely to be inaccurate. That said, biases that can 
appear in self-report data are expected to be minimal in the present study, since the parameters that have been analyzed mostly did not refer to driver opinions. Still, future studies need to be undertaken which include objective measures of system use.

The findings of the present study represent an interesting starting point for strategies that aim at supporting the regular use of speed regulation assistance. Although CC and SL use are related to different factors, that need to be addressed separately, the results also suggest that measures fostering the use of in-vehicle technologies more in general are likely to increase the use and perceived safety benefit of speed regulation systems.

\section{ACKNOWLEDGMENTS}

The research leading to these results has received funding from the European Community's 7th Framework Programme (FP7/2007-2013) under grant agreements 218560 and 238833.

\section{REFERENCES}

Bonnard, A., Karmann, N., Lancelle, V. and Brusque, C. (2009). Terminology definition and selection of In-Vehicle Technologies to investigate. Report FP7 Project INTERACTION.

Britschgi, V. and Rämä, P. (2010). Survey on individual and cross-cultural differences in the use of In-Vehicle Technologies. Report FP7 Project INTERACTION.

European Commisson [EC](2006). Use of Intelligent Systems in Vehicles. Special Europbarometer 267 /Wave 65.4 - TNS Opinion \& Social. EC, December 2006.

International Transport Forum [ITF] (2008). Towards Zero - Ambitious Road Safety Targets and the Safe System Approach. International Transport Forum. OECD Publishing, 14 Oct 2008.

Lheureux, F., Saad, F., Pianelli, C., Abric, J.-C. and Roland, J. (2006). Behavioural changes due to long term use of speed limiter and cruise control. AIDE project - Adaptive Integrated Driver-vehicle Interface, 2006, D 1.2.4, 39-64.

Peden, M., Scurfield, R., Sleet, D., Mohan, D., Hyder, A.A., Jarawan, E. and Mathers, C. (2004). World Report on Road Traffic Injury Prevention. Geneva: World Health Organization.

Pereira, M., Bruyas, M.P., Kaufmann, C., Britschgi, V., Diez Gi, J.L., Zaoral, A. (in press). Reported use of speed control systems: cruise control and speed limiter. IET Intelligent Transport Systems.

Várhelyi, A. and Mäkinen, T. (2001).The effects of in-cars speed limiters: field studies. Transportation Research Part C, 9, 191-211.

Vollrath, M., Schleicher, S. and Gelau, C. (2011). The influence of Cruise Control and Adaptive Cruise Control on driving behaviour - A driving simulator study. Accident Analysis and Prevention, 43, 1134-1139.

Young, K. L. and Regan, M. A. (2007). Use of manual speed alerting and cruise control devices by car drivers. Safety Science, 45(4), 473-485. 